\title{
A IMPORTÂNCIA DO “ESTAR AQUI”: DIMENSÕES ESPACIAIS DA PROXIMIDADE GEOGRÁFICA NA PESQUISA CIENTÍFICA EM REDE
}

\author{
Allison Bezerra Oliveira. \\ Universidade Estadual da Região Tocantina do Maranhão - UEMASUL \\ Curso de Geografia, Imperatriz, MA, Brasil \\ Grupo de Pesquisas Socioeconômicas do Maranhão - GPS \\ allisonbzr@gmail.com
}

Ana Cristina de Almeida Fernandes

Universidade Federal de Pernambuco - UFPE

Curso de Geografia, Recife, PE, Brasil

Grupo de Pesquisa em Inovação, Tecnologia e Território anacf.ufpe@gmail.com

\begin{abstract}
RESUMO
A revolução das tecnologias de informação, processamento e comunicação tem possibilitado instantaneidade comunicacional nunca antes vista: de informações, sons e ideias. Tal perspectiva tem sido apropriada por diversos segmentos da sociedade e com diversos objetivos, o de multiplicar a produção do conhecimento é um entre tantos. A produção do conhecimento científico em rede com pessoas em espaços geográficos distantes tem sido assim compreendida como forte mecanismo de superação das desigualdades. Em meio a essas questões, o presente trabalho tem como objetivo analisar a importância da proximidade geográfica na pesquisa científica em rede mediante os contornos tecnológicos comunicacionais. Para tal, empreendeu-se reflexão teórica sobre o tema, mapeou-se as relações de pesquisa em rede no estado de Pernambuco na área de Farmácia como recorte metodológico, construiu-se um mapa de fluxos dessas relações e aplicou-se questionários com os pesquisadores. Os resultados obtidos apontam para a importância do "estar aqui" como elemento mais eficaz que a comunicação instantânea na troca de informações na pesquisa científica.
\end{abstract}

Palavras-chave: Proximidade Geográfica. Pesquisa em Rede. Comunicação Instantânea. Pernambuco.

\section{THE IMPORTANCE OF "BEING HERE": SPACE DIMENSIONS OF GEOGRAPHICAL PROXIMITY IN SCIENTIFIC RESEARCH IN NETWORK}

\begin{abstract}
The revolution of the information, processing and communication technologies created the possibility to instantly communicate as never before: sending information, sounds and ideas. This perspective has been used by several parts of the society with several goals like propagation of knowledge and others. The creation of scientific knowledge without the geographical barriers, are well understood as strong mechanism to overcome inequalities. Among these questions, this article has the following goals: study how important the geographic proximity inside the scientific research is when using all the network communication technologies available. To achieve this goal, we will use a theoretical thinking about the subject with the use of Pharmacology research relationship network inside the state of Pernambuco, using questionnaires with the researchers in order to build a communication flow between them. The results pointed out the matter of "being here" as the most efficient element in the instant communication in order to exchange information in the scientific research.
\end{abstract}

Keywords: Geographic Proximity. Network research. Instant Communication. Pernambuco. 


\section{INTRODUÇÃO}

A revolução tecnológica tem possibilitado gradativamente ao longo dos anos a realização de maior mobilidade generalizada: das pessoas, da energia, da informação, das ideias, dos bens, dos usos, dos produtos e dos serviços. Tal mobilidade tem possibilitado instantaneidade, diminuição das noções tempo-espaciais e assegurado aos centros de decisão um real poder sobre os outros pontos do espaço.

Tal processo, que corresponde à própria natureza do capitalismo, acelerou-se para hoje alcançar o seu ápice, com a predominância, em toda parte, de um único sistema técnico, ou, nas palavras de Santos (2006, p. 128), de uma "unicidade técnica" que é base material da globalização. Com a emergência do meio técnico-científico-informacional, o atual sistema técnico se tornou comum a todas as civilizações, culturas e sistemas políticos, a todos os continentes e lugares, mesmo que sua difusão e inserção ocorram em escalas desiguais (SANTOS, 2006).

Tal unicidade técnica tem proporcionado o que Santos (2006) chama de unicidade de momentos, e Harvey (2008) chama de compressão tempo-espacial. Em ambas as reflexões, é inegável a participação efetiva das tecnologias de informação, processamento e comunicação. A informação ganhou a possibilidade de fluir instantaneamente, sem nenhuma defasagem, comunicando para quase todos os lugares. Embora seja desigual, tal processo desencadeou aumento significativo e gradativo nos processos sociais e relacionais; a expressão máxima dessas transformações é a expansão das redes.

O debate das redes chega a ser onipresente quando falamos nos contornos atuais possibilitados pelos avanços tecnológicos da comunicação e do transporte. A lógica viária de uma sociedade em rede baseada em fluxos tem proporcionado gradativa diminuição de algumas rugosidades espaciais e expressivas reflexões sobre a rigidez física da distância. Tais transformações têm sido apropriadas pela pesquisa científica sob a compreensão de que 0 trabalho coletivo amplifica os resultados à medida que a troca de informações se torna mais profícua em conjunto do que entre indivíduos isolados. Nesse sentido, as telecomunicações são a base material para o estabelecimento de intercâmbio científico entre pessoas que antes estavam distantes geograficamente.

Contudo, embora inegáveis as contribuições proporcionadas pelas telecomunicações e a telemediação eletrônica nos processos de cooperação e pesquisa científica em rede, muitas reflexões têm ao longo dos últimos anos apresentado concepções de que tais tecnologias estariam cada vez mais diminuindo a geograficidade dos processos sociais cotidianos, das rugosidades, das proximidades. Em outras palavras, estariam substituindo qualidades formais do espaço geográfico, como a proximidade geográfica.

Nesse contexto, o presente artigo apresenta uma análise teórico-conceitual de tais processos, além de refletir sobre a relevância ou não da proximidade geográfica nos processos de pesquisa científica em rede, mesmo com o advento tecnológico. Dessa forma, pretende-se elucidar uma questão principal: considerando-se as possibilidades permitidas pelo desenvolvimento técnico-comunicacional e o financiamento científico, a proximidade geográfica ainda é elemento relevante na pesquisa científica em rede?

Além desta breve introdução, este trabalho está assim dividido: a primeira conexão, "Redes, telecomunicações e mediação eletrônica", em que se apresenta o debate teórico-conceitual sobre as redes, as telecomunicações e parte dos desdobramentos por elas possibilitados, seguida da segunda conexão, "A pesquisa científica em rede e suas contribuições", conceituando o objeto de estudo deste trabalho e sua inserção no Brasil e no mundo como mediador de resultados e benefícios, e a terceira conexão, "Questões sobre a importância da proximidade geográfica e do 'estar aqui'", que visa reiterar a importância de qualidades formais do espaço, tais como a proximidade geográfica. Apresenta-se ainda tópico metodológico com a discussão dos resultados em seção intitulada "Evidências da importância do 'estar aqui' na pesquisa científica em rede", além das considerações finais. 


\title{
REDES, TELECOMUNICAÇÕES E MEDIAÇÃO ELETRÔNICA
}

Dias (2003, p. 147) destaca que uma rede apresenta a propriedade de conexidade, isto é, através da conexão de seus nós, ela, simultaneamente, tem a potencialidade de solidarizar ou de excluir, de promover a ordem e a desordem. Além disso, a autora evidencia que a rede é uma forma particular de organização social, técnica ou natural, e, no âmbito dos processos de integração, desintegração e exclusão espacial, "aparece como instrumento que viabiliza [...] duas estratégias: circular e comunicar".

A reflexão sobre os atuais contornos das redes (ancorada na estrutura tecnológica e informacional) aponta diretamente para a concepção da "sociedade em rede" de Manuel Castells, segundo a qual a sociedade moderna é caracterizada pela predominância da forma organizacional da rede em todos os campos da vida social.

\begin{abstract}
A sociedade em rede, em termos simples, é uma estrutura social baseada em redes operadas por tecnologias de comunicação e informação fundamentadas na microeletrônica e em redes digitais de computadores que geram, processam e distribuem informação a partir de conhecimento acumulado nos nós dessas redes. (CASTELLS, 1999, p. 20).
\end{abstract}

Para Castells (1999), Lemieux e Ouimet (2004) e Portugal (2007), as razões do sucesso da utilização do conceito de rede, fundamentalmente, estariam ligadas ao desenvolvimento das tecnologias de informação, processamento e comunicação, possibilitando a existência de conexões onde antes havia isolamento. Para Castells (2006, p. 18), "as redes de comunicação digital são a coluna vertebral da sociedade em rede, tal como as redes de potência (ou redes energéticas) eram as infraestruturas sobre as quais a sociedade industrial foi construída".

Nesse contexto, o paradigma da mediação eletrônica é a base material - sistema de objetos para a expansão penetrante de redes tecnológicas na estrutura social da sociedade contemporânea. Conforme Castells (1999, p. 497): "As redes constituem a nova morfologia social de nossas sociedades, e a difusão da lógica de redes modifica de maneira substancial a operação e os resultados dos processos produtivos e de experiência, poder e cultura".

As redes de mediação tecnológica não apenas distribuem poder, mas também tornam possível a disseminação de novas e diferentes formas de poder: senhas, servidores, firewalls, grupos online, comunidades... Assim, conforme Tinland (2001), as redes estruturam, à sua maneira, 0 campo de forças das relações de cooperação e de antagonismo que estão presentes na sociedade humana. As redes "são, de fato, instrumentos de poder e de rivalidades para seu controle. Elas são suscetíveis [...] de funcionar como instrumentos de integração e de exclusão, na linha direta dos processos de diferenciação" (TINLAND, 2001, p. 263).

Enquanto as instituições territoriais tradicionais são mais hierárquicas e rígidas, a internet tende a privilegiar modos de relacionamento transversais e estruturas mais fluidas, em maior sintonia com as estruturas de redes, que caracterizam os processos sociais e políticos nas sociedades democráticas modernas, como aponta Frey (2003). Nesse sentido, "interações acontecem com diversas pessoas, conexões mudam entre múltiplas redes e hierarquias podem ser reduzidas e recursivas" (WELLMAN, 2001, p. 227).

As redes facilitam a melhoria do desempenho, isto é, um aumento dos resultados produzidos, além de possibilitarem a autonomia dos parceiros e aumentarem sua capacidade de aprendizagem (FREY, 2003). De acordo com o tipo de rede e o objetivo da atividade a ser desenvolvida, a instantaneidade e a praticidade proporcionam a atuação de vários atores de diversos níveis transversais em uma mediação tecnológica, que deve ser considerada como importante suporte telecomunicacional. Webconferências, suporte para mensagens instantâneas, download, anexação de arquivos, disponibilidade de tutoriais, fóruns de dúvidas, formação de grupos virtuais para debates e transferência de informações contribuem significativamente em determinados momentos do trabalho em rede para o acesso à informação. 
Não há dúvidas de que as redes eletrônicas transformam as dimensões de tempo e espaço. A informação é transmitida em tempo real e pode-se estabelecer contatos imediatamente, independentemente da distância espacial. A comunicação em rede garante, em princípio, um acesso universal, confortável, não-filtrado e de baixo custo a informacões e processos políticos. Entretanto, o potencial democrático específico da internet baseia-se em sua estrutura não-hierárquica e cibernética que, em princípio, favorece a interatividade. (FREY, 2003, p. 177).

As redes eletrônicas digitais podem ser simultaneamente adaptáveis e híbridas graças à sua capacidade de descentralizar o seu desempenho e conectividade ao longo de uma rede de componentes autônomos, enquanto se mantêm as capacidades de coordenar todo o processo de determinada atividade de forma descentralizada, com a possibilidade de partilhar a tomada de decisões em vários pontos por uma parcela do grupo ou por todo ele, em momentos distintos ou simultâneos, dinamizando os resultados e procedimentos metodológicos de processos de trabalho, "endereçamento por centro de interesse e a comunicação todos-todos são condições favoráveis ao desenvolvimento de processos de inteligência coletiva" (LÉVY, 1998, p. 44)

Atores têm a oportunidade de se conectarem às suas redes instantaneamente, vislumbrando sons, imagens e interagindo todos ao mesmo tempo. Ao contrário das conexões estabelecidas pelo telégrafo, por exemplo, onde os nós da rede eram apenas pontos em uma continuidade geográfica, as redes eletrônicas permitem a construção e transmissão de inúmeras particularidades sociais - os pontos já não são mais pontos, são pessoas - e, até certa medida, a transmissão desses aspectos ajuda significativamente na manutenção da interatividade por todos os nós.

A comunicação de mão dupla, que se torna possível na internet, contrapõe-se à comunicação de mão única, que prevalece nos meios de comunicação de massa. Enquanto, por exemplo, na televisão os debates públicos são conduzidos em geral pelos líderes de opinião, sem uma participação ativa dos cidadãos comuns, surgem com a internet novas possibilidades de criação de uma esfera pública interativa, um tipo de "ágora eletrônica". (ROESLȨR, 1997, p. 182).

A imprensa, a edição, o rádio e a televisão funcionam segundo um esquema em estrela, ou "um para todos". Um centro emissor envia mensagens na direção de receptores passivos e, sobretudo, isolados uns dos outros. Mas não há reciprocidade nem interação (ao menos no interior do dispositivo), e o contexto é imposto pelos centros emissores (LÉVY, 1998). O emissor acaba tendo um papel central na transmissão de informação não pela capacidade de interação com os vários indivíduos, mas pela incapacidade destes de interagirem, expressarem-se, e, consequentemente, transformarem-se em emissores também.

O correio e o telefone desenham um esquema em rede, ponto a ponto, "um para um", no qual, ao contrário da mídia tradicional, as mensagens podem ser endereçadas com precisão e, sobretudo, trocadas com reciprocidade. Entretanto, esse esquema em rede não cria comunidade ou "público", pois a partilha de um contexto em escala é significativamente difícil e o tempo de espera entre uma troca e outra, às vezes, pode ser longo ou pouco difuso.

Já a telemediação eletrônica por meio das inúmeras tecnologias de processamento, informação e comunicação combina vantagens dos dois sistemas anteriores, permitindo ao mesmo tempo a reciprocidade na comunicação e a partilha de um contexto entre os vários membros da rede que o utilizam. Tal dinâmica tem sido apropriada por diversos atores nas mais distintas atividades, inclusive na pesquisa científica.

\section{A PESQUISA CIENTÍFICA EM REDE E SUAS CONTRIBUIÇÕES}

Se a produção de um novo conhecimento necessita primordialmente do intercâmbio de ideias e de que a informação circule entre os vários pares envolvidos, nada mais apropriado que a estrutura no formato de redes seja utilizada pela pesquisa científica, uma vez que esta, como já mencionado em tópico anterior, viabiliza duas estratégias básicas, a circulação e a comunicação, como aponta Dias (2003). Tal possibilidade de colaboração - calcada ainda mais nas tecnologias comunicacionais - aponta para transformações expressivas na produção do conhecimento científico. 
A produção de conhecimento novo requer, cada vez mais, o esforço compartilhado entre pares, inclusive aqueles situados em contextos nacionais e institucionais diversos, em torno de ações colaborativas e iniciativas conjuntas para o encontro de soluções a questões e problemas comuns no mundo globalizado. A cooperação internacional deverá levar em conta essa tensão entre o acirramento da competitividade e a necessária produção colaborativa de conhecimento. (MACIEL; ALBAGLI, 2010, p. 9).

Para Adams (2012), as novas redes estão reforçando a competência e a capacidade das economias emergentes de investigação e alterando o equilíbrio global da atividade de pesquisa científica que, por sua vez, pode revelar diferentes formas de abordar os problemas das grandes instituições mundiais.

[...] abrem-se novas oportunidades de uma possível democratização do acesso à informação e ao conhecimento e sua apropriação social em favor de um desenvolvimento em novas bases, sob diferentes pontos de vista. No plano da geopolítica, coloca-se o desafio da desconcentração do conhecimento, tanto entre países quanto entre regiões. (MACIEL; ALBAGLI, 2010, p. 9)

De forma meramente quantitativa, podemos observar os resultados desses processos evolutivos de pesquisa científica em rede no trabalho de King (2012), em que o autor faz um retrospecto da quantidade de autores por trabalho científico com base em dados da Nature. No início de 1980, os artigos com mais de 100 autores eram raros; em 1990, o registro anual com esse número ultrapassou 500. O primeiro trabalho com 1.000 autores foi publicado em 2004, e uma pesquisa com 3.000 autores, em 2008. No ano passado, um total de 120 artigos de física teve mais de 1.000 autores, e 44 tinham mais de 3.000. Muitos deles são de colaborações no Large Hadron Collider (LHC) do CERN, na Europa, no laboratório de aceleração de partículas, perto de Genebra, na Suíça.

Nesse contexto, Gama e Velho (2005, p. 205) observaram dados do Science and Engineering Indicators (2004) sobre a cooperação internacional entre pesquisadores, destacando que houve um aumento dessa parceria, conforme pontuam:

A cooperação internacional em ciência e tecnologia tem crescido a uma taxa significativa. Partindo de uma base inexpressiva em meados do século $\mathrm{XX}$, a cooperação internacional representa hoje uma parcela considerável da pesquisa científica. Quando medida, por exemplo, através da contagem de artigos publicados em co-autoria por pesquisadores trabalhando em, pelo menos, dois países diferentes, a cooperação internacional mais do que duplicou entre 1988 e 2001 - passou de 8 para 18\% do total de artigos publicados e indexados pelo Science Citation Index.

Tais dados, apontam para uma compreensão há muito clara nos países de ponta da pesquisa científica mundial: de que o trabalho cooperativo amplifica os resultados na medida em que agentes com conhecimentos complementares se integram na busca de um objetivo comum. $E$ este pode ser potencializado em empreendimentos estruturados no formato das redes científicas com suporte nas tecnologias de processamento, informação e comunicação.

Kodama (1992) afirma que alguns dos mais significativos avanços científicos surgiram da integração ou fusão de campos científicos anteriormente separados ou onde havia pouco diálogo entre seus pesquisadores. Porém, como aponta Fujino et. al. (2009, p. 215-216),

A ciência é uma atividade eminentemente coletiva e social, construída por meio de relações sociais entre os pares. Seus resultados (avanços ou retrocessos) são resultantes da interação entre os pesquisadores, seja de maneira direta (por meio de desenvolvimento de projetos e publicações em coautoria) ou indireta (por meio da citação de trabalhos da comunidade científica). De certo modo, existe uma relação entre todos os membros de uma comunidade científica de determinada área, tanto nacionalmente quanto internacionalmente, e, em consequência ao desenvolvimento da atividade científica como um todo.

Para Lara e Lima (2009), a cooperação científica é um processo social intrínseco às formas de interação humana para efetivar a comunicação e o compartilhamento de competências e

$\begin{array}{llllll}\text { Caminhos de Geografia } & \text { Uberlândia - MG } & \text { v. 20, n. } 69 & \text { Março/2019 } & \text { p. 467-483 } & \text { Página } 471\end{array}$


recursos. Para as autoras, a pesquisa científica em rede é um meio para otimizar recursos, dividir trabalho, aliviar o isolamento próprio da atividade acadêmica e criar sinergia entre os membros dessa atividade. A rede científica, de acordo com Oliveira (2014, p. 131), pode ser considerada como

\begin{abstract}
Agrupamentos de pesquisadores e suas afiliações e grupos, em uma continuidade de pesquisa em rede, com vários níveis hierárquicos ou não, em espaços geográficos distintos ou não, onde se tem por finalidade a construção de laços de cooperação, confiança, reciprocidade e compartilhamento de experiências, promovendo, assim, o progresso da ciência por meio da produção de bens, serviços, conhecimentos e inovações.
\end{abstract}

Para Funaro (2010, p. 53), "na pesquisa científica os atores são cientistas, e o vínculo entre eles é a colaboração, que posteriormente resultará em uma publicação em forma de colaboração ou coautoria". A publicação ou registro de uma patente, por exemplo, é a expressão dos resultados e objetivos aspirados por uma rede científica.

Tal compreensão é tão importante pelo fato de que, historicamente, um dos grandes gargalos e empecilhos para a emancipação e o desenvolvimento brasileiro se traduz na sua capacidade ainda limitada de produzir ciência e tecnologia. A partir de 2004, inseriu-se o estímulo à pesquisa científica em rede como estratégia eficaz de obtenção de resultados mais expressivos e céleres pelas políticas de Ciência, Tecnologia \& Inovação (OLIVEIRA; FERNANDES, 2015).

Para a sustentação e o fortalecimento do Esforço Nacional em Ciência, Tecnologia e Inovação, as principais estratégias são: "§ 63. J) Esforços para criação de parcerias e redes de pesquisas nos países da América Latina, África e Ásia, visando a enfrentar problemas comuns" (BRASIL, 2008, p. 22). Além disso, acrescenta-se "em médio prazo: estímulo ao desenvolvimento de redes de cooperação técnico-científica; c) Em longo prazo: a produção de conhecimentos por meio de redes de cooperação" (BRASIL, 2008, p. 24-26).

Para a superação das desigualdades regionais: "§ 94 Estimular a criação de redes de cooperação" (BRASIL, 2008, p. 29-30). Dentro do âmbito do Plano de Ação em Ciência, Tecnologia e Inovação (PACTI 2007-2010; 2011-2014; 2015-2018): "promover a formação de redes de pesquisa em todo território nacional". Tais redes proporcionaram o aumento de intercâmbio de ideias e troca de informações, promovendo maior produção de conhecimento nacional que viesse a ser utilizado em áreas estratégias para o país.

Essa estratégia [da pesquisa em rede] visa a ampliar a capacidade de produzir conhecimentos para qualificar as decisões no âmbito da gestão pública. Dessa forma, será possível suprir uma das maiores necessidades nas sociedades modernas, que é dispor de informações técnicas e científicas indispensáveis para fundamentar o processo de tomada de decisão, que tem forte impacto sobre diversos campos científicos e contribui para 0 estabelecimento de um novo patamar nas relações entre ciência, Estado e sociedade. (BRASIL, 2008, p. 30).

Neste contexto, as agências de fomento brasileiras em especial o CNPq e CAPES passaram a compreensão clara de que o incentivo e apoio à formação de redes de pesquisa é extremamente eficaz para a indução ao desenvolvimento de uma dada área do conhecimento. Assim, o fortalecimento da capacidade instalada nas instituições de pesquisa e da realização de pesquisa colaborativa e interdisciplinar, com integração entre as várias ciências, possibilitando a troca de metodologias, promoção do intercâmbio de dados, bem como a produção conjunta e a divulgação científica dos resultados seria expressivamente otimizado. Desta forma, a articulação de redes científicas inter-regionais e interdisciplinares de pesquisa, estimularia o intercâmbio entre instituições que concentram as mais diversas competências, e promoveria a interação entre pesquisadores, o uso otimizado de recursos, o compartilhamento de infraestrutura para a pesquisa, com a perspectiva de convergência dos resultados.

Tal compreensão se difundiu e serviu como suporte efetivo de promoção da pesquisa científica em rede nos últimos anos no Brasil. Mas será que tal entendimento aliado ao desenvolvimento técnico-comunicacional tem promovido efetivamente o aumento da inserção de pesquisadores 
situados em localidades geográficas distantes? A proximidade geográfica ainda é componente valorizado? O debate apresentado a seguir nos dá indicativos pertinentes sobre o tema a partir de importante literatura.

\title{
SOBRE A IMPORTÂNCIA DA PROXIMIDADE GEOGRÁFICA E DO “ESTAR AQUI”
}

Antes de apresentarmos resultados da pesquisa, cabe empreender uma reflexão teóricoconceitual acerca da discussão sobre a importância da proximidade na ciência geográfica. As ideias aqui apresentadas corroboram com os debates desenvolvidos por autores como Gertler (1995); Allen (2000); Storper e Venables (2001); Torre e Rallet (2005); Lagendijk e Lorentzen (2007).

O primeiro ponto fundamental é o que consideramos como proximidade geográfica. A "proximidade" aqui mencionada é usada tanto no sentido literal, no que se refere à distância geográfica, como no sentido de compartilhamento de língua comum, modos de comunicação, estrutura de trabalho, costumes, convenções, simbolismos e normas sociais.

Ou seja, a proximidade como continuidade social, dentro de um contexto histórico e geográfico constante de (re)produção das relações sociais cotidianas. Do ponto de vista das construções e produções acessíveis e cotidianas, Lagendijk e Lorentzen (2007, p. 460) destacam que fundamentalmente a proximidade geográfica não deve ser equacionada apenas como proximidade física, pura e simplesmente.

\begin{abstract}
Pelo contrário, é um conceito dinâmico, intimamente associado com a noção de estruturação espacial. Por um lado, a proximidade geográfica é um produto de uma construção histórica, de um acumulado de infraestruturas de transporte e de locais de encontro, tanto de uma forma e sentido mais material e virtual, bem como, gostaríamos de acrescentar, a formação de espaços territorialmente delimitados, dimensões sociais, institucionais, políticas e econômicas. (LAGENDIJK; LORENTZEN, 2007, p. 460).
\end{abstract}

Para Lagendijk e Lorentzen (2007), a proximidade geográfica promove e potencializa também efeitos casuais não esperados, além de sustentar a conectividade e posicionalidade dos agentes. Da mesma forma, Gertler (1995) destaca a importância espacial como componente na facilitação do processo de compartilhamento de códigos de comunicação e cooperação, bem como na construção de um legado comum de práticas e instituições sociais. Ainda segundo o autor, a proximidade parece facilitar a formação e manutenção em alta qualidade de relacionamentos que necessitem de intensiva interação e proporciona maior e mais livre fluxo de informações entre os atores.

Para ele, no caso em que grandes distâncias venham a intervir, problemas podem daí decorrer, mesmo com a funcionalidade eletrônica. A "falta de proximidade" tenderia a tornar o processo de cooperação oneroso ${ }^{1}$; e mesmo (in)voluntariamente os membros ainda tendem a procurar 0 estabelecimento de conexões com outros que estão próximos.

As ideias de Gertler (1995) são reforçadas por Lagendijk e Lorentzen (2007, p. 462) ao destacarem que "os lugares continuam sujeitos a formas mais simbólicas de posicionalidade". $\mathrm{Na}$ mesma linha de raciocínio, Allen (2000) evidencia que as rotinas organizacionais em curso e as práticas sociais de trabalhos coletivos envolvidos em empreendimentos comuns são mais facilmente fortalecidas pelas interações cotidianas. Para tal, as práticas espaciais produzem perspectivas que em alguns casos - ou momentos - são permitidas à distância, embora mantenham significativa importância presencial.

Portanto, estar presencialmente em determinados momentos com os membros de uma rede não só facilita a interação, a coordenação e a comunicação entre os membros, como

\footnotetext{
1 O autor indica alguns problemas tais como: fuso horário, postos de fronteiras, distâncias físicas de deslocamento, diferenças entre infraestruturas técnicas, problemas de comunicação relacionados às diferentes linguagens, idiomas, regionalismos, diferenças contextuais das práticas no local de trabalho, as culturas de formação etc.
} 
potencializa os níveis de proximidade social e, consequentemente, uma maior troca e compreensão de códigos e signos, além do estabelecimento de laços de confiança. A necessidade de contatos face a face é tanto mais importante quanto maior for o caráter tácito do conhecimento, por exemplo.

[...] com relação à troca eletronicamente mediada, a estrutura do contato face a face oferece possibilidade incomum de interrupção, reparo, feedback e aprendizagem. Contrariamente a interações grandemente sequenciais, a interação face a face possibilita a duas pessoas emitir e receber mensagens simultaneamente. O ciclo de interrupção, feedback e reparo, possível na interação face a face é tão rápido que é praticamente instantâneo. (NOHRIA; ECCLES, 1992, p. 292).

Polanyi (1987, p. 4) afirma que "sabemos mais do que somos capazes de comunicar". A comunicação face a face extrai sua riqueza e poder não apenas por nos permitir estarmos em contato presencialmente e detectarmos mensagens intencionais, mas também mensagens não intencionais que podem ser carregadas apenas por esse contato visual.

O contato FaF, elimina o anonimato, faz aumentar a probabilidade de boas escolhas, graduais e interativas, sobre as habilidades dos outros, quando não se pode saber, de início, a respeito de sua capacidade de operosidade; também pode fazer com que os indivíduos se tornem mais capazes de sinalizar para os outros suas habilidades e níveis de operosidade. É uma maneira de tornar transparente e de baixo custo este tipo de informação, embora também permita, nos primeiros contatos FaF, que certas pessoas se juntem e permaneçam no grupo. (STORPER; VENABLES, 2001, p. 41).

Da mesma forma, Polanyi (1987) argumenta que o conhecimento científico é produzido por indivíduos que impregnam sua busca por novos conhecimentos com conteúdos profundamente pessoais, que são resultado de uma série de interações e particularidades. Em outras palavras, o conhecimento científico não seria apenas um conjunto claramente articulado de axiomas, regras, algoritmos e declarações. Estes, por sua vez, em alguns casos, não podem ser compreendidos através de telefonemas, chats, webconferências, e-mails, servidores e demais tecnologias.

Ou seja, mesmo com os meios eletrônicos, o conhecimento não flui tão facilmente. Isso porque a sua transmissão é mais comum por meio da interação face a face entre os parceiros que já compartilham questões espaciais "básicas": a mesma língua, "códigos" comuns de comunicação, convenções e normas compartilhadas, o conhecimento pessoal de cada um baseado em uma história passada de colaboração de sucesso ou interação informal. Essas semelhanças são ditas para servir ao propósito vital de construção de confiança entre os parceiros, o que, por sua vez, facilita o fluxo local de conhecimento tácito entre os parceiros (GERTLER, 1995; MORGAN, 2006).

Torre e Rallet (2005, p. 53) também discutem que mesmo com a atual explosão comunicacional, ou "a onipresença promovida pela comunicação e mobilidade - física e virtual", certos recursos e relações permanecem espacialmente enraizados. Afirmam ainda que a proporção de enraizamento da presencialidade de contatos face a face varia de acordo com a atividade, "mas que em todas, ainda encontraríamos faísca da necessidade da produção espacial presencial cotidiana".

[...] com relação à troca eletronicamente mediada, a estrutura do contato face a face oferece possibilidade incomum de interrupção, reparo, feedback e aprendizagem. Contrariamente a interações grandemente sequenciais, a interação face a face possibilita a duas pessoas emitir e receber mensagens simultaneamente. O ciclo de interrupção, feedback e reparo, possível na interação face a face é tão rápido que é praticamente instantâneo. (NOHRIA; ECCLES, 1992, p. 292).

Portanto, a proximidade virtual proporcionada pelos fluxos tecnológicos não pode substituir a proximidade geográfica nas transações de alta complexidade e ambiguidade, por exemplo, não

$\begin{array}{llllll}\text { Caminhos de Geografia } & \text { Uberlândia - MG } & \text { v. 20, n. } 69 & \text { Março/2019 } & \text { p. 467-483 } & \text { Página } 474\end{array}$


só no âmbito do próprio funcionamento da pesquisa científica, mas na própria construção e consolidação de relações que necessitem de circunstâncias presenciais.

A presença mútua - estar suficientemente próximo, a ponto de tocar-se um no outro - permite o "contato" visual e a "proximidade emocional", a base para a construção das relações humanas. A comunicação FaF, mais que apenas uma troca, é um desempenho pelo qual o discurso e outros tipos de ações e contextos se juntam para exercitar a comunicação, de uma maneira muito complexa, em muitos e diferentes níveis ao mesmo tempo. (STORPER; VENABLES, 2001, p. 32).

Para eles, a possibilidade dos pesquisadores "estarem aqui" e se comunicarem face a face em dados momentos, não só é necessário, como reduz uma série de incertezas, e dinamiza o trabalho cooperativo. Em outras palavras, ter os demais membros "próximos" na hora de se formar uma equipe ou durante a realização do projeto facilita a realização do trabalho em si, além da inserção de novos membros no grupo. Os dados apresentados a seguir reforçam as colocações aqui apresentadas e nos sugerem cautela às considerações acerca de elementos espaciais da proximidade geográfica.

\section{MATERIAIS E MÉTODOS}

$\mathrm{Na}$ busca de validação da literatura sobre o tema que destaca a relevância do "estar aqui" utilizou-se mecanismos da pesquisa em Ciência da Informação, análise de redes sociais, além da própria Geografia. Focou-se inicialmente, na utilização de informações contidas em publicações científicas e técnicas, envolvendo essencialmente artigos, livros ou patentes, uma vez que estas são fontes materiais confiáveis de produção de conhecimento. Dessa forma, utilizou-se a grande área da Saúde e, em específico, a subárea de Farmácia, como recorte de amostragem para a pesquisa. A escolha da área de Farmácia deu-se por dois motivos principais: a) pela importância dessa área na superação de desigualdades sociais no âmbito da saúde; e b) por tal área ser considerada na literatura evolucionária sobre conhecimento e inovação tecnológica (PAVITT, 1984) como essencialmente dependente da troca de conhecimentos com outras áreas em sua pesquisa científica básica, como Biologia, Química, Física, Biomedicina, Medicina, Engenharia, Biotecnologia, dentre outras.

Posteriormente, utilizou-se o censo do CNPq de 2014 para identificação do quantitativo de grupos de pesquisa e pesquisadores da grande área de Farmácia no estado de Pernambuco². Eliminando-se as repetições, chegou-se ao número de 24 líderes e 156 pesquisadores cadastrados na base de dados. Foram verificados os artigos que incluíam tais pesquisadores como (co)autores, que fossem oriundos de pesquisas em rede e publicados e evidenciados na Plataforma Lattes. Para maior amplitude de informações utilizou-se três bases de dados indexadas: ISI Web of Knowledge, SciVerse Scopus e Scientific Electronic Library Online SciELO. O intuito foi também mapear autores que não aparecessem em grupos de pesquisa, mas que desenvolvessem pesquisas em rede. Excluindo-se novamente todas as repetições, chegou-se a um volume de 259 pesquisadores no ano em questão.

Essas informações foram transformadas em matrizes no Excel $(259 \times 259)$ para a construção de uma matriz que é reflexo numérico das relações expostas nos artigos científicos, e esta, por sua vez, serve como modelo para que a rede seja ao final mapeada. Após a construção da matriz numérica, os dados são migrados para os softwares Ucinet $^{3}$ e Netdraw que criam o desenho final. Tal processo foi desenvolvido a partir da metodologia trabalhada por Newman (2001), aperfeiçoado pelos pesquisadores da ciência da informação e pode ser melhor detalhado na figura 1 a seguir.

\footnotetext{
2 Diante do volume de dados gerados por uma pesquisa a nível nacional, utilizou-se apenas uma Unidade Federativa brasileira como amostragem, o que, por sua vez, já gerou quantitativo expressivo de informações.

${ }^{3}$ O Ucinet é um pacote de software para a análise de dados de redes sociais. Foi desenvolvido por Lin Freeman, Everett Martin e Steve Borgatti e inclui a ferramenta de visualização da rede NetDraw.
} 
Figura 1 - Processo de mapeamento de rede científica com base em artigos publicados
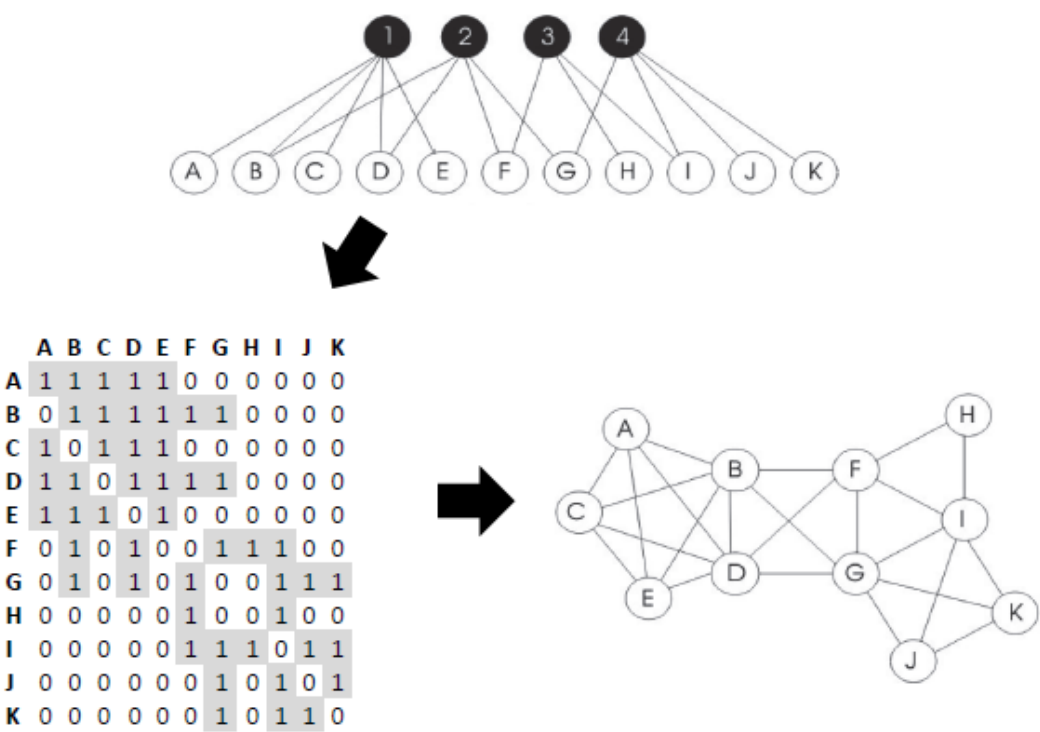

Fonte - Elaboração própria (2014) a partir de Newman (2001) e Balancieri et. al. (2005).

Nela, os números de 1 a 4 são os artigos publicados, e os pesquisadores são as letras compreendidas entre $\mathrm{A}$ e $\mathrm{K}$, a partir das várias conexões entre os vários autores por meio de suas relações na pesquisa científica, gera-se uma matriz relacional, onde 1 significa que existe conexão entre dois atores, e 0 que não existe conexão. A matriz é o reflexo numérico das relações expostas nos artigos científicos, ela por sua vez, serve como modelo para que a rede seja ao final mapeada.

Os dados do mapeamento das relações da rede científica foram condensados e transformados posteriormente em um mapa de fluxos dessas conexões apresentando a localização geográfica dessas interações no mundo. Tais informações foram consolidadas com questionários eletrônicos enviados aos principais pesquisadores que aparecem na pesquisa, obteve-se $67 \%$ de retorno dos questionários.

\section{EVIDÊNCIAS DA IMPORTÂNCIA DO “ESTAR AQUI" NA PESQUISA CIENTÍFICA EM REDE}

É plausível que, no processo de mapeamento de uma rede científica que tem Pernambuco como ponto de partida, se encontre uma predominância de membros desse estado. Contudo, é de se considerar que sejam encontrados pesquisadores de diversos espaços geográficos, uma vez que, como já abordado neste trabalho, são inegáveis as contribuições das telecomunicações e o próprio incentivo político para a formação de redes.

Entretanto, para além dessas questões, a rede expressa na Figura 2 nos sugere outras informações pertinentes. A primeira delas trata da concentração de pesquisadores localizados em Pernambuco (pontos vermelhos e azuis); ao todo, 75\% (195 pesquisadores) de todos os agentes envolvidos estão no estado, mais do que isso, estão concentrados na Região Metropolitana da capital Recife ${ }^{4}$ (RMR).

\footnotetext{
${ }^{4}$ É comum ainda ser encontrada expressiva concentração geográfica da pesquisa científica na Região Metropolitana de Recife. O estado de Pernambuco passou por um processo desigual de distribuição das instituições de pesquisa e ensino superior, e o movimento de interiorização é relativamente recente.
} 


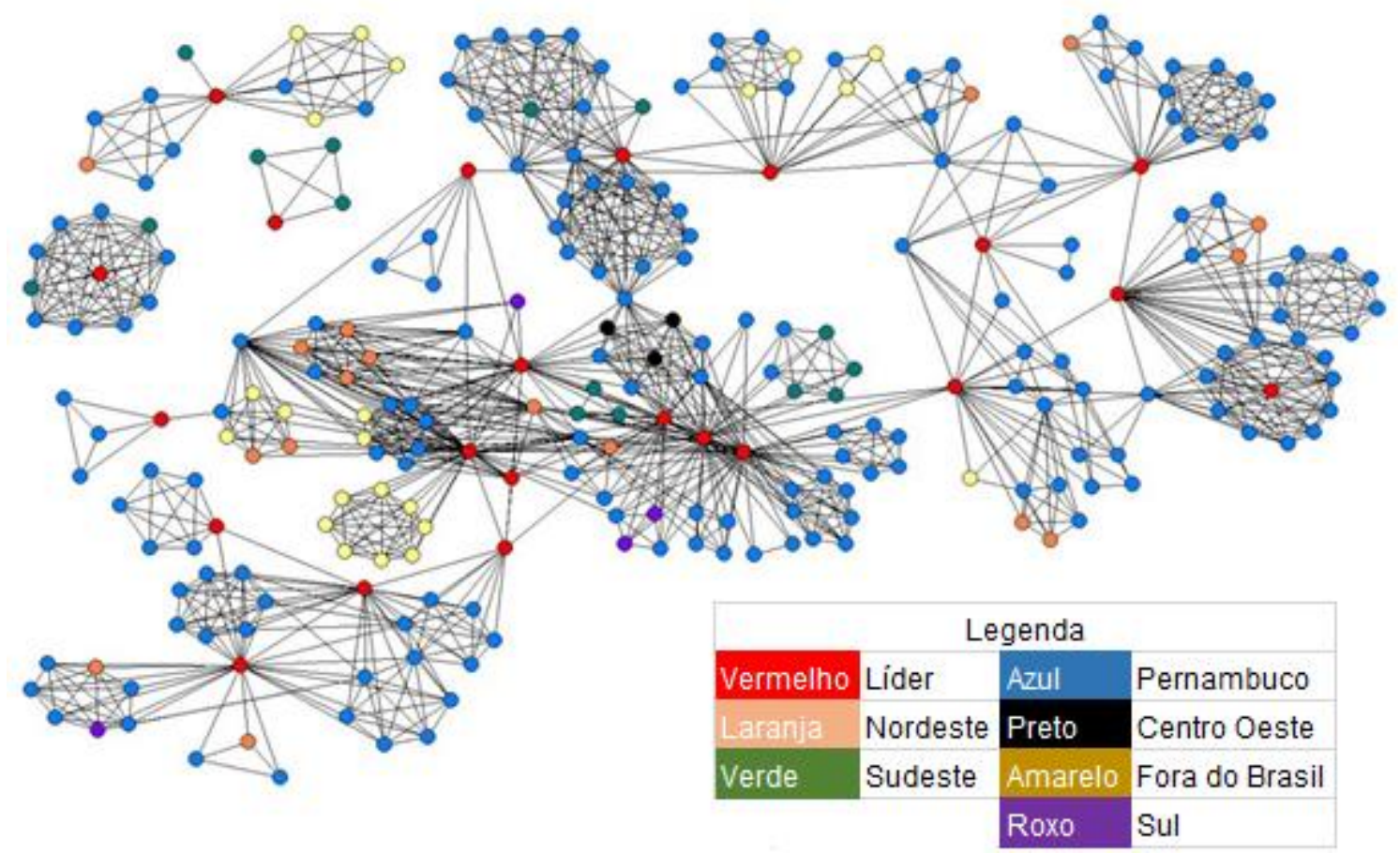

Fonte - Oliveira (2014)

As relações estabelecidas são quase que exclusivamente em formato circular, em torno dos líderes dos grupos de pesquisa (pontos vermelhos), o que indica que o sistema de objetos da produção científica na área de Farmácia é significativamente fixo no estado e tem seu funcionamento em torno dos grupos de pesquisa (que detém além do suporte técnico para a pesquisa o espaço corriqueiro de contato presencial). Estes se tornam elementos palpáveis e cotidianos na construção das relações de pesquisa, mesmo que em diversos momentos sejam utilizados mecanismos eletrônicos para a comunicação e a delegação de tarefas.

Em apenas três pequenas redes não há conexão com o maior aglomerado científico, as relações se formam quase que exclusivamente na Universidade Federal de Pernambuco e são também enormemente dependentes dos líderes dos grupos de pesquisa (pontos vermelhos) e os laboratórios. A cooperação nesse caso é fundamental para o processo de pesquisa e publicação final de um novo princípio ativo ou fármaco, desta forma, é justificável a formação da estrutura da rede e as conexões quase em sua totalidade.

É importante destacar que nas redes mais densas construídas em torno dos líderes dos grupos de pesquisa há uma predominância de atores exclusivamente fincados no estado de Pernambuco. Há apenas uma rede formada predominantemente com pesquisadores fora do Brasil. Tal informação será evidenciada no mapa de fluxos discutido a seguir.

A concentração geográfica de pesquisadores, mais que fruto de uma necessidade de proximidade geográfica no processo de pesquisa pernambucano na área de farmácia nos mostra que ela é fundamental para que as relações de cooperação na pesquisa possam consolidar, para isso, ações e objetos cotidianos, fixos e móveis mas que estejam a serviço dessa dinâmica estejam acessíveis. Em outras palavras, os líderes de grupo de pesquisas que detêm o capital de financiamento além dos laboratórios são membros fundamentais para a permanência e a circulação de pessoas nesse emaranhado de conexões.

Quando olhamos a participação quantitativa de pesquisadores de outros estados, temos 19 (8\%) em outros estados da região Nordeste, 3 (1\%) estão na região Centro-Oeste, $12(5 \%)$ no Sudeste, $8(3 \%)$ no Sul do Brasil, e $22(8 \%)$ estão em outros países. Todos os pesquisadores 
encontrados nas regiões Nordeste e Centro-Oeste executaram atividades no passado no estado de Pernambuco e construíram vínculos profissionais duradouros. Tais conexões podem ser vistas sob o formato de um mapa de fluxos, expresso na Figura 3.

Figura 3 - Mapa de fluxos de pesquisadores.

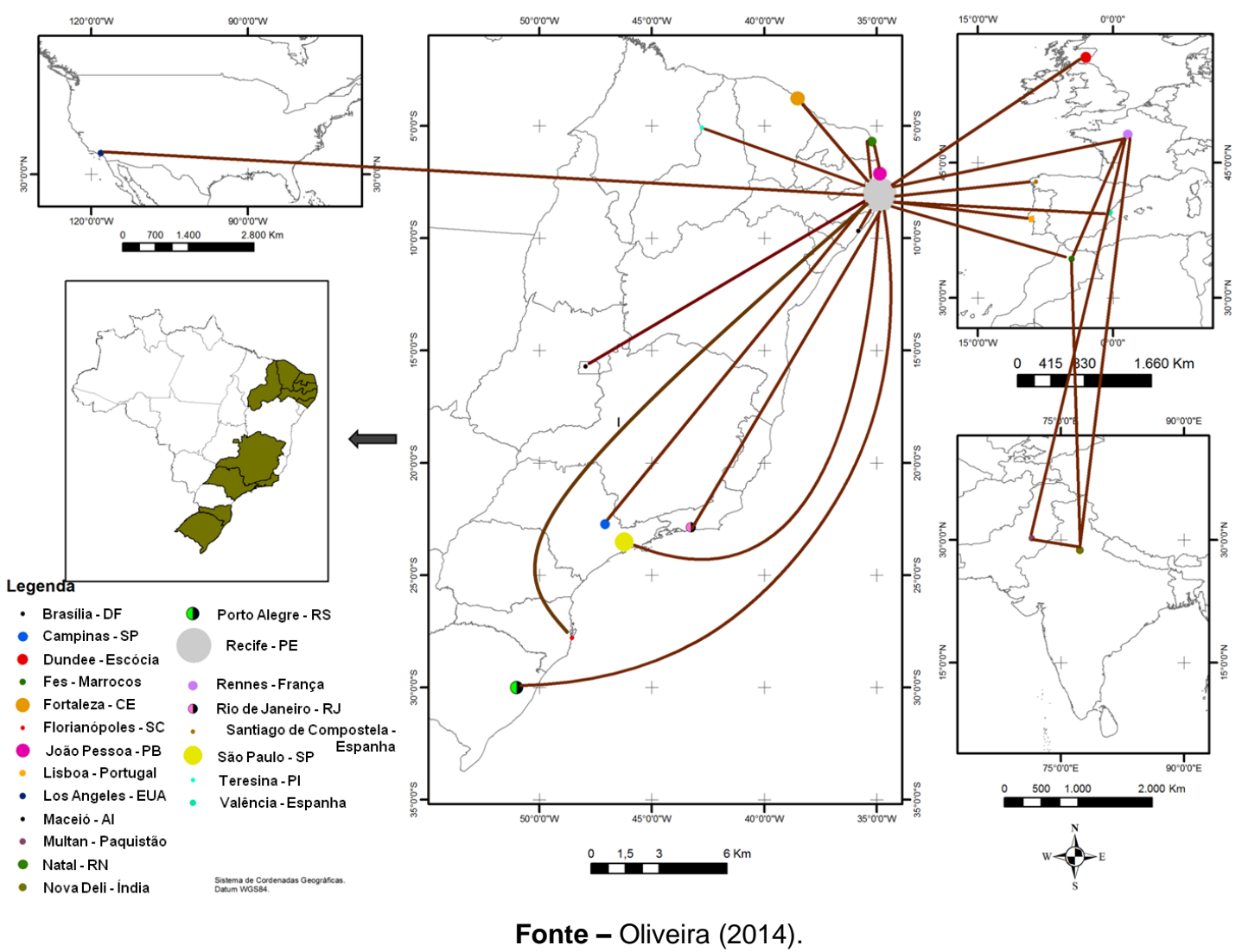

A concentração na região Nordeste fica em torno de $83 \%$, o que é um percentual expressivo se compararmos com a participação de atores em outras regiões. Das 22 conexões fora do país, 8 delas foram estabelecidas por brasileiros que se encontravam em outros países, restando 14 pesquisadores efetivamente estrangeiros, que se conectaram apenas com 3 pesquisadores pernambucanos, em outras palavras: com três grupos de pesquisa apenas.

As concentrações geográficas apresentadas nos dados apontam para uma proximidade que ultrapassa a mera distância em quilômetros e diz respeito à concentração em um mesmo estado e à grande centralidade apresentada pelos líderes dos grupos de pesquisa e suas instalações. Apontam também para o fato de que ainda são mais valorizadas interações e cooperações não planejadas - frequentes e eficazes - mais amplas, tais como compartilhamento de idioma comum, regionalismos, estruturas e regimes de trabalhos, convenções e outros simbolismos mais facilmente apreendidos por quem "está aqui". A própria distância é colocada também como elemento importante quando se refere aos membros que estão em outros estados no Nordeste.

A fim de podermos compreender com mais propriedade os dados, para nós, assim como para Gertler (1995), a forma mais direta de tentarmos determinar a importância do "estar aqui" no trabalho científico em rede em meio às telecomunicações - além da própria construção de uma literatura pertinente sobre a temática - é perguntarmos diretamente para os agentes em questão.

Nesse caso, buscou-se verificar qual a importância atribuída pelos pesquisadores ao fato de ter outros membros "próximos" na hora de se estabelecer uma pesquisa científica em rede ou mesmo realizar a própria pesquisa. A proximidade em questão, como já mencionada, diz 
respeito tanto à perspectiva de ter acesso contínuo e talvez cotidiano ao outro membro, permitir que se tenha acesso a este de maneira mais rápida e fácil, quanto ao estabelecimento de proximidade física pactuado cotidianamente pela construção de convívios espaciais contínuos. Os principais questionários estão compilados no Quadro 1 a seguir:

Quadro 1 - Questões sobre a importância da proximidade geográfica.

\begin{tabular}{|c|c|c|c|}
\hline Questionamento & Sim & Não & Irrelevante \\
\hline Sobre a importância de conhecer pessoalmente o pesquisador & $65 \%$ & $30 \%$ & $5 \%$ \\
\hline Sobre a importância de ter trabalhado anteriormente com o outro & $85 \%$ & $15 \%$ & \\
\hline Sobre a importância de relações orientador $x$ orientando & $95 \%$ & & $5 \%$ \\
\hline Sobre a importância de encontros presenciais na pesquisa em rede & $70 \%$ & $30 \%$ & \\
\hline $\begin{array}{l}\begin{array}{l}\text { Se considera que a comunicação eletrônica é substituta eficaz da comunicação } \\
\text { presencial }\end{array} \\
\end{array}$ & $30 \%$ & $70 \%$ & \\
\hline Sobre a importância de pesquisar com quem está próximo geograficamente & $95 \%$ & & $5 \%$ \\
\hline
\end{tabular}

$$
\text { Fonte - Oliveira (2014). }
$$

Quando perguntados sobre a importância de se conhecer pessoalmente o pesquisador, a importância de ter trabalhado previamente com o outro na hora de se estabelecer uma pesquisa em rede e a importância da relação orientador $x$ aluno, em todas as perguntas mais de $60 \%$ responderam "sim", afirmando se tratarem de fatores importantes. Outro ponto pertinente trata das relações de confiança entre orientador $x$ orientando, que possibilitam a construção de relações de confiança contínuas a partir da formação acadêmica; 95\% dos pesquisadores informaram valorizar tais elementos, e apenas $5 \%$ consideraram irrelevante.

Essas respostas ratificam os formatos circulares da rede científica mapeada, onde os pesquisadores em sua maioria se relacionam em torno do líder do grupo de pesquisa e dos laboratórios. Reforçam também as informações indicadas pelos fluxos, onde $71 \%$ dos 64 atores que não se encontravam em Pernambuco já haviam estabelecido pesquisas no estado.

Nesses casos a proximidade geográfica é mais que meramente física, é uma proximidade estabelecida pelas relações de confiança que são mais facilmente constituídas cotidianamente. Para os pesquisadores, essas rugosidades permitidas apenas pelo exercício contínuo e presencial de cooperação são fundamentais para o ingresso e a permanência de membros nas diversas redes estabelecidas. Além de serem fundamental para o próprio exercício satisfatório da pesquisa científica.

Quando perguntados sobre a importância de encontros presenciais na pesquisa em rede, $70 \%$ dos entrevistados afirmaram valorizar esse tipo de encontro. Nessa mesma percepção espacial do trabalho em rede, $70 \%$ dos entrevistados não considera a comunicação eletrônica como substituta eficaz da comunicação presencial. Há, dessa forma, uma inequívoca e clara valorização do contato face a face, que somente é possibilitado pelo "estar aqui". Tais perspectivas são finalizadas com o significativo percentual de $95 \%$ de respostas positivas quanto à importância de se pesquisar com quem está próximo geograficamente.

\section{CONSIDERAÇÕES FINAIS}

O "estar aqui" que promove a comunicação face a face possibilita a redução de incertezas e dinamiza o processo cooperativo. Essa especificidade da proximidade geográfica se relaciona com certas modalidades do exercício de confiança cotidiana, que têm a ver não só com a 
reputação do outro (o que poderia se realizar, até certo ponto, à distância), mas com práticas mais complexas e mais efetivadas em longo prazo. Essa prática constitutiva da confiança se apoia nas relações que só podem ser realizadas pessoalmente.

A pesquisa científica em rede desta forma não pode ser compreendida apenas como um elemento dual: ancorado no suporte tecnológico e no fomento financeiro. As relações sociais conduzidas nas rugosidades cotidianas ainda mantêm importância expressiva dentro do convívio e formação diária. A concentração geográfica de pesquisadores reforçou isso.

Desta forma, a proximidade geográfica abrange as mesmas contradições que produzem 0 espaço e que também geram as condições de reprodução das relações sociais. Estas são fortalecidas na medida em que os atores envolvidos estão presencialmente "à disposição" para trocas simbólicas, políticas, científicas e econômicas cotidianas.

A importância simbólica da proximidade pode ser reiterada nos questionários a medida em que pode se perceber que relações de confianças, frutos de vínculos de orientação e formação acadêmica eram extremamente valorizadas onde mesmo pesquisadores que estavam em outros países colaborando já haviam exercido anteriormente atividades cooperativas, sendo a experiência importante elemento na permanência de membros nos grupos.

As discussões acerca das comunicações eletrônicas devem ser relativizadas com mais cautela e olhadas sob múltipla variáveis e espaços geográficos. Isso implica considerarmos que a imersão tecnológica além das práticas cooperativas são frutos de contextos geográficos distintos, são frutos de temporalidades, científicas, técnicas e sobretudo políticas e devem ser olhadas não de uma forma generalista, mas sim, extremamente específica.

Por fim, é importante ratificar que existem nuanças da comunicação pessoal que requerem proximidade entre as pessoas, como regionalismos, contextos históricos e linguagens corporais promovidas apenas pelo contato face a face. É imprescindível, portanto, não confundir o alcance espacial com a profundidade das relações sociais, assim como os contextos geográficos distintos, uma vez que o entendimento de linguagens regionais e a difusão de conhecimento em rede - telecomunicações - podem não ser facilmente compreendidos.

\section{AGRADECIMENTOS}

Agradecimentos à Fundação de Amparo à Ciência e Tecnologia do Estado de Pernambuco FACEPE pela bolsa de doutoramento concedida que foi fundamental para a realização desta pesquisa.

\section{REFERÊNCIAS}

ADAMS, J. The rise of research networks. Nature, Londres, v. 490, p. 335-336, 18 out. 2012. https://doi.org/10.1038/490335a

ALLEN, J. Power/economic knowledge: symbolic and spatial formations. In: BRYSON, J. et. al. (Org.). Knowledge, Space, Economy. London: Routledge, 2000, p. 15-33.

BALANCIERI, R. et. al. Análise de redes de colaboração científica sob as novas tecnologias de informação e comunicação: um estudo na Plataforma Lattes. Ci. Inf., Brasília, v. 34, n. 1, p.64-77, jan./abr. 2005 https://doi.org/10.1590/S0100-19652005000100008

BRASIL. 2ª Conferência Nacional de Ciência, Tecnologia e Inovação em Saúde. Brasília:

$\begin{array}{llllll}\text { Caminhos de Geografia } & \text { Uberlândia - MG } & \text { v. 20, n. } 69 & \text { Março/2019 } & \text { p. 467-483 } & \text { Página } 480\end{array}$


Ministério da Saúde, 2004.

Ministério da Saúde. Política Nacional de Ciência, Tecnologia, Inovação em Saúde (PNCTIS). 2. ed. Brasília: Editora do Ministério da Saúde, 2008. Disponível em: <http://bvsms.saude.gov.br/bvs/publicacoes/Politica_Portugues.pdf>. Acesso em: 02 abr. 2018.

CASTELLS, M. A Sociedade em Rede: do Conhecimento à Política. In A sociedade em rede do conhecimento à Ação Política. (Orgs.) CASTELLS, M. \& CARDOSO, E. Imprensa Nacional de Portugal, 2006.

A sociedade em rede - A era da informação: economia, sociedade e cultura. Vol. 1. 6. ed. São Paulo: Paz e Terra, 1999.

DIAS, L. C. Redes: emergência e organização. In: CASTRO, I. E. de; GOMES, P. C. da C.; CORREAA, R. L. (Org.). Geografia: conceitos e temas. 5. ed. Rio de Janeiro: Bertrand Brasil, 2003.

FREY, K. Desenvolvimento sustentável local na sociedade em rede: o potencial das novas tecnologias de informação e comunicação. Rev. Sociol. Polít., Curitiba, n. 21, p. 165-185, nov. 2003.

https://doi.org/10.1590/S0104-44782003000200011

FUJINO, A. et. al. Políticas públicas de incentivo à formação de redes sociais e colaborativas em ciência e tecnologia. In: POBLACIÓN, D. A.; MUGANAINI, R.; RAMOS, L. M. S. V.

C.(Org.). Redes sociais e colaborativas em informação científica. São Paulo: Angellara, 2009.

FUNARO, V. M. B. de O. Rede colaborativa entre autores em Odontologia: docentes dos programas de pós-graduação credenciados em universidades participantes do Sistema de Informação Especializado na Área de Odontologia (SIEO). 2010. 184 f. Tese (Doutorado em Ciência da Informação) - Escola de Comunicações e Artes, Universidade de São Paulo, São Paulo, 2010.

GAMA, W.; VELHO, L. A cooperação científica internacional na Amazônia. Estudos Avançados, São Paulo, v. 19, n. 54, p. 205-224, ago. 2005.

https://doi.org/10.1590/S0103-40142005000200012

GERTLER, M. S. "Being there": proximity, organization, and culture in the development and adoption of advanced manufacturing technologies. Economic Geography, Worcester, v. 71, 1 26, 1995.

https://doi.org/10.2307/144433

HARVEY, D. Condição Pós-Moderna. 17. ed. São Paulo: Edições Loyola, 2008.

KING, C. Multiauthor Papers: Onward and Upward. ScienceWatch, Philadelphia, v. 23, p. 1-2, July 2012. Disponível em: <http://go.nature.com/w3f2jt>. Acesso em: 18 fev. 2018.

KODAMA, F. Technology fusion and the new R\&D. Harvard Business Review, Boston,

p. 70-78, July/Aug. 1992.

LAGENDIJK, A.; LORENTZEN, A. Proximity, Knowledge and Innovation in Peripheral Regions. On the Intersection between Geographical and Organizational Proximity. European Planning

Studies, v. 15, n. 4, Apr. 2007. 


\section{https://doi.org/10.1080/09654310601133260}

LARA, M. L. G.; LIMA, V. M. A. Termos e conceitos sobre redes sociais colaborativas. In: POBLACIÓN, D. A. de; MUGANAINI, R.; RAMOS, L. M. S. V. C. (Org.). Redes sociais e colaborativas em informação científica. São Paulo: Angellara, 2009.

LEMIEUX, V.; OUIMET, M. L'Analyse structurale des réseaux sociaux. Québec: Les Presses de l'Université Laval; Bruxelles: De Boeck Université, 2004.

https://doi.org/10.3917/dbu.lemie.2004.01

LEVY, P. A Revolução contemporânea em matéria de comunicação. Tradução: SILVA, J. M. da. Revista FAMECOS, Porto Alegre, v. 5, n. 9, p. 37-49, dez. 1998. https://doi.org/10.15448/1980-3729.1998.9.3009

MACIEL, M. L.; ALBAGLI, S. Cooperação internacional em ciência e tecnologia: desafios contemporâneos. In: CENTRO DE GESTÃO E ESTUDOS ESTRATÉGICOS. Cooperação Internacional na Era do Conhecimento. Brasília, 2010, p. 9-22.

https://doi.org/10.1590/S0101-20612010000400005

MORGAN, K. The Exaggerated Death of Geography: Learning, Proximity and Territorial Systems. Journal of Economic Geography, v. 4, n. 1, p. 3-21. London: Oxford University Press, 2006.

https://doi.org/10.1093/jeg/4.1.3

NEWMAN, M. E. J. Scientific colaboration networks I. Network construction and fundamentals results. Physical Review E, Woodbury, v. 64, 2001. https://doi.org/10.1103/PhysRevE.64.016131

NOHRIA, N.; ECCLES, R. Networks and organizations: structure, form and action. Boston: Harvard Business School Press, 1992.

OLIVEIRA, A. B. A importância do espaço geográfico na construção e funcionamento de redes científicas na área de farmácia em Pernambuco fomentadas pela Política Nacional de Ciência, Tecnologia e Inovação em Saúde (PNCTIS). 2014. 346 f. Tese (Doutorado em Geografia) Universidade Federal de Pernambuco, Recife, 2014.

OLIVEIRA, A. B.; FERNANDES, A. C. A. Mudanças recentes nas políticas de ciência, tecnologia, inovação para a saúde no Brasil: considerações sobre o fomento à pesquisa em rede. In: ENCONTRO NACIONAL DA ANPEGE, 11., Presidente Prudente. Anais eletrônicos... Presidente Prudente: ANPEGE, 2015. Disponível em:

<http://www.enanpege.ggf.br/2015/anais/arquivos/12/391.pdf>. Acesso em: 18 fev. 2018.

PAVITT, K. Sectoral Patterns of Technical Change: Towards a Taxonomy and a Theory. Research Policy, Amsterdam, North-Holland, v. 13, n. 6, p. 343-373, Dec. 1984. https://doi.org/10.1016/0048-7333(84)90018-0

POLANYI, M. The Tacit Dimension. In: PRUSAK, L. Knowledge in Organizations. Nova lorque: Harper \& Row, 1987.

PORTUGAL, S. Contributos para uma discussão do conceito de rede na teoria sociológica. Oficina do CES, Coimbra, n. 271, 2007.

ROESLER, A. Bequeme Einmischung. Internet und Öffentlichkeit. In: MÜNKER, S.;

ROESLER, A. (Org.). Mythos Internet. Frankfurt am Main: Suhrkamp, 1997. 
SANTOS, M. A natureza do espaço: técnica e tempo, razão e emoção. 4. ed. São Paulo: EDUSP, 2006.

STORPER, M.; VENABLES, A. O burburinho: a força econômica da cidade. In: DINIZ, C.; LEMOS, M. (Org.). Economia e território. Belo Horizonte: UFMG, 2001.

TINLAND, F. Interactions, réseaux, différenciation. In: PARROCHIA, D. (Org.). Penser les réseaux. Seyssel: Éditions Champ Vallon, 2001. p. 245-265.

TORRE, A. \& RALLET, A. Proximity and localization. Regional Studies, Brighton, v. 39, p. 4759, 2005.WELLMAN, B. Physical Place and Cyberplace: The Rise of Personalized Networking. International Journal of Urban and Regional Research, Oxford, v. 25, n. 2, p. 227-252, June. 2001.

https://doi.org/10.1111/1468-2427.00309

Recebido em: 07/04/2018

Aceito para publicação em: 20/08/2018 